\title{
Role of Serology and Histopathology in Diagnostic of Human Cystic Echinococcosis
}

\section{Albadawi AAM1*, Saad MBE ${ }^{2}$, Alzain $\mathrm{SM}^{3}$, Osman NTM44, Gaber $\mathrm{AH}^{5}$, Abdelmonem $\mathrm{M}^{6}$, Osman $\mathrm{HB}^{7}$ and Ahmed $\mathrm{ME}^{8}$ \\ ${ }^{1}$ Department of Parasitology, National Public Health Laboratory (NPHL), Republic of} Sudan

2Department of Parasitology, Faculty of Medical Laboratory Science, Omdurman Ahlia University, Republic of Sudan ${ }^{3}$ Department of Parasitology, National Public Health Laboratory (NPHL), Republic of Sudan ${ }^{4}$ Nawal Tagelsir Mohammad Osman ${ }^{5}$ Department of Mycology, Faculty of Medicine, University of Khartoum, Republic of Sudan ${ }^{6}$ Department of Mycology, Faculty of Medicine, University of Khartoum, Republic of Sudan ${ }^{7}$ Department of National Public Health Laboratory (NPHL), Ministry of Health; Republic of Sudan ${ }^{8}$ Department of National Public Health Laboratory (NPHL), Ministry of Health; Republic of Sudan

*Corresponding author: Adam Alfaki Mohammad Albadaw, Department of Parasitology, National Public Health Laboratory (NPHL), Ministry of Health, Republic of Sudan, Tel: 249122710595; Email: adama027@gmail.com

\section{Abstract}

Introduction: Hydatid disease is an important emerging neglected disease worldwide, with significant geographic variation in seroprevalance. The disease is commonly diagnosed on basis of clinical suspicion, imaging and serology. This study was conducted to evaluate our experience with serology in diagnosing hydatid disease in terms of identifying the numbers of patients testing positive for antibodies against Echinococcus, and to study their clinical profile. This study included all patients who tested for anti-Echinococcus IgG antibodies by ELISA in the years 2011-2013. The clinical characteristics of seropositive patients were contrasted with seronegative patients, and the test results were correlated with radiological findings and, where performed, with histopathological studies. Analysis was performed on SPSS. Out of 31 patients evaluated by serology for Echinococcosis during the study period, 7 (22.58\%) were seropositive. Liver was the most frequent site involved (24 patients; $77.77 \%$ ). Second organ the lung $5(16.13 \%)$ bone $1(3.23 \%)$ and the optic 1(3.22\%).Of the 29 patients who also underwent biopsy, all the seven patients with positive biopsy were seropositive, suggesting $100 \%$ sensitivity. The specificity was $83.33 \%$, but this might be a lower estimate due to potentially high rate of false-negative biopsies, as all the seropositive patients also had imaging features suggestive of disease. The positive and negative predictive values were $77.77 \%$ and $90.90 \%$, respectively. ELISA for detection of anti Echinococcus antibodies is a simple serological test that helps in correlation with imaging finding in the diagnosis and subsequent management of hydatid disease. 
Material and Methods: A cross sectional study was done on biological material (obtained from haptic and lung hydatid cysts). The viability criteria used were ovoid form invaginated scolices and intact calcareous corpuscles the presences of vibrating movements; and the absence of "vital" staining. The cysts were grouped according to diameter cysts from small cysts to very large size cysts. Descriptive statics for the collection of the prevalence of fertility; analytical statistics for comparison of groups, and multivariate analysis for examination of the association between cyst fertility and clinical variables. Is using to carry out by SPSS programme.

Result: Out of 31 patients evaluated by serology and histopathology for Echinococcosis during the study period, 7 $(22.58 \%)$ were seropositive. Liver and lung was the most frequent site involved all the seven patients with positive biopsy were seropositive, suggesting $100 \%$ sensitivity. The specificity was $83.33 \%$, but this might be a lower estimate due to potentially high rate of false-negative biopsies, as all the seropositive patients also had imaging features suggestive of disease. The positive and negative predictive values were $77.77 \%$ and $90.90 \%$, respectively.

Conclusion: The serodiagnosis correlated with radiological finding and where performed with histopathological studies. And the evaluation ELISA test in sensitivity and specificity, prevalence rates of the fertility of hydatid cysts and viability of protoscoleces and diversity sites location observed in human.

Keywords: Human Cystic Echinococcosis; Microscopy; ELISA and histopathology; Sudan

\section{Introduction}

Hydatidosis is a cycle-zoonotic parasitic infection caused by the Cestodes Echinococcus granulosus [1]. It is an important pathogenic, zoonotic and parasitic infection (acquired from animals) of humans, following ingestion of tapeworm eggs excreted in the faeces of infected dogs. It is a major endemic health problem in certain areas of the world $[2,3]$. Cystic echinococcosis is globally distributed and found in every continent except Antarctica; Echinococcosis, WHO [4]. Iceland and Greenland; The disease is characterized by an almost worldwide distribution causing significant morbidity and considerable socioeconomic impact in highly endemic regions; Significantly reducing meat and milk production as well as causing fertility loss in livestock [5-8]. It is especially prevalent in parts of Eurasia, north and east Africa, Australia, and South America, Communities that practice sheep farming experience the highest risk to humans, McManus DP, et al. [9]. But wild animals can also serve as an avenue for transmission. For example, dingoes serve as a definitive host before larvae infect sheep in the mainland of Australia [9]. Sled dogs may expose moose or reindeer to E. granulosus in parts of North America and Eurasia. The incidence rate in hospitals is about 1000 times less than the prevalence as only a small proportion of patients who develop severe symptoms seeks medical care [10]. As per the recommendation by the World
Health Organization, hospital data can be used to measure the prevalence of $C E$ in a population [11-13]. The presence of these isolated cases in man which is not really normal intermediate host for the hydatid cyst but still having our attention to the importance of hydatid cyst, which could be considered as that occurs in wild animals which are becoming relatively more important as that to human health [14].

The disease appears as multiple solid, tumor-like cysts sprouting on the interior and exterior of the organs. The cysts can range in size from 2 to $20 \mathrm{~cm}$, are filled with fluid and can contain many smaller, daughter cysts [15]. The fertility of cysts is an important factor that can influence the transmission of E. granulosus. Depending on the nature of infected hosts and the sites of infection, cysts may have different fertility rates. These cysts commonly occur in the lungs and liver, but can be found in any other organ or tissue including bones, spleen, heart, eye, brain, and genitourinary tract. Anatomic clinical changes are peculiar to localization in the bone [16]. From the anatomopathologic stand point, this localization marks the torpid, insidious progression of the parasite into the bone tissue, leading to a diffuse, extensive, invasive process; so from the clinical stand point, wherever it is localized, its complete surgical eradication is rarely possible [16]. 
Hepatic cysts were more frequent than pulmonary cysts at ratio/or symptomatic cases $[17,18]$. The factors that determine the final anatomic location of cysts are still unknown; however the liver is the organ most frequently infected because oncospheres penetrate the intestinal wall and tend to disseminate to the liver via the portal vein. Variation in cyst location frequencies observed in diverse studies throughout the world could be due to infection with different species Pierangeli, et al. [19]. The germ membrane has less resistance to trauma, presents itself with a great number of nuclei and has a double role: helps the parasite reproduce and secretes the cyst's liquid. This layer permits also the development of "daughter vesicles". (Figure 1)The hydatid sand found in the clear liquid has the immunological capacity of response, and both can generate the anaphylactic reaction of the host Tenguria RK, Naik MI, et al. [20].

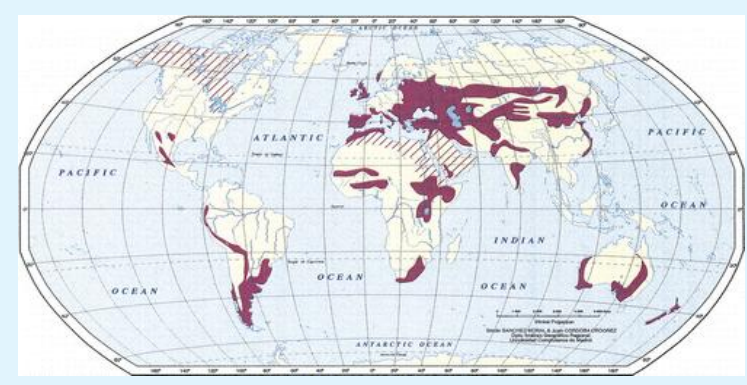

Figure 1: Geographic distribution of hydatid disease Map shows areas in which hydatid disease is endemic due to the transmission of $E$ granulosus by means of the dog-sheep cycle (solid red areas).

Red stripes indicate areas where transmission occurs by means of alternative life cycles in which carnivores such as wolves and foxes serve as definitive hosts and goats, camels, and horses serve as intermediate hosts. Transmission by means of alternative life cycles is common in North Africa, the Middle and Far East, the United States, Canada, and Iceland. E. granulosus is transmitted from the intermediate host (sheep) to the definitive host (dogs) by frequent feeding of offal, also referred to as "variety meat" or "organ meat". Consuming offal containing E. granulosus can lead to infection; however, infection is dependent on many factors.

The lifecycle (figure 2) of E. granulosus involves dogs and wild carnivores as a definitive host for the adult tapeworm [2]. Definitive hosts are where parasites reach maturity and reproduce. Wild or domesticated ungulates, such as sheep, serve as an intermediate host $[2,21]$. Transitions between life stages occur in intermediate hosts. The larval stage results in the formation of echinococcal cysts in intermediate hosts [2]. Echinococcal cysts are slow growing [2]. But can cause clinical symptoms in humans and be life-threatening [9]. Cysts may not initially cause symptoms, in some cases for many years. Symptoms developed depend on location of the cyst, but most occur in the liver, lungs, or both [9]. When contaminated waste is excreted into the environment, intermediate host has the potential to contract the parasite by grazing in contaminated pasture, perpetuating the cycle [21,22]. Granulosus is transmitted from the intermediate host (sheep) to the definitive host (dogs) by frequent feeding of offal, also referred to as "variety meat" or "organ meat". Consuming offal containing E. granulosus can lead to infection; however, infection is dependent on many factors [9].

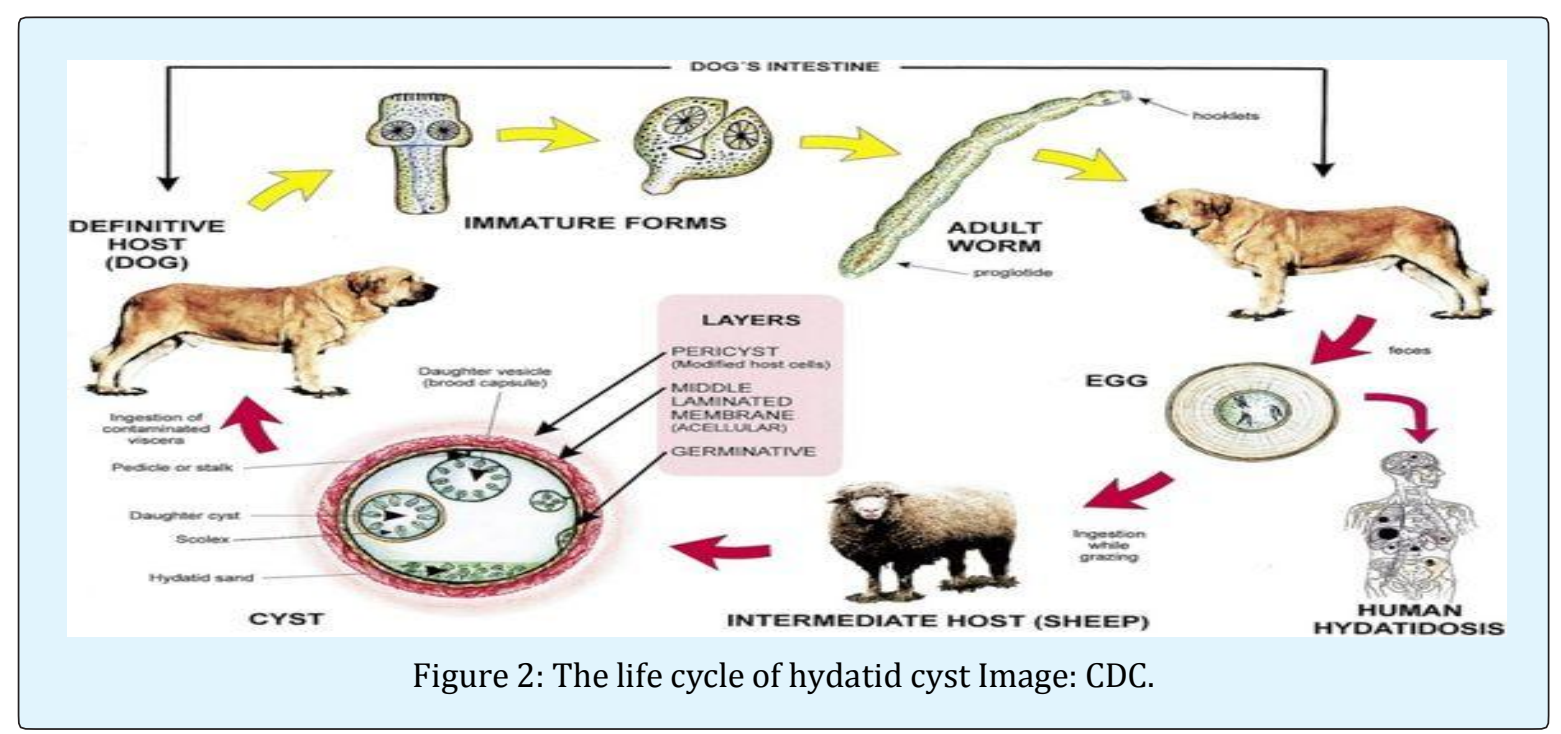

Albadawi AAM, et al. Role of Serology and Histopathology in Diagnostic of Human Cystic Echinococcosis. J Inf Dis Trav Med 2018, 2(3): 000116. 


\section{Journal of Infectious Diseases \& Travel Medicine}

\section{Rationale}

Hydatidosis is known to occur in Sudan in man as well as animals but the extent of the disease in human population is not known. This is mainly due to the nonspecific clinical presentation and the nonexistence of sensitive and specific diagnostic methods, a part from confirmation at operation. Therefore this study was undertaken to study epidemiology of the disease among human population and to extract antigenic constituents from hydatid cysts in man, and attempt to analyse them and use them as targets for different serological tests for the laboratory diagnosis of hydatidosis in man.

\section{Objective}

- To evaluate the sensitivity and specificity of ELISA test for detection of hydatid disease

- To carry out the fertility of the cyst and viability of protoscoleces

\section{Specific Objectives}

- To collect the cysts from internal organs (liver, lungs and other o rgans); and aspirate the fluid by centrifugation to separate the protoscoleces and large particles from the fluid for serology diagnosis.

- Macroscopic and microscopic examination for viable or non viable

- Protoscoleces

- To confirm histological examination the nature of a suspected hydatid cyst with the serology and imaging techniques.

\section{Materials and Methods}

\section{Study Design}

It is a cross sectional study, in biological material (the fluid from internal organ of the body of the human e.g. liver \& lung).

\section{Study Area}

The study was conducted at the hospitals of Khartoum state from November 2011- July 2013.

\section{Study Period}

This study included an analysis of suspected cases of hydatid disease which were tested for presence of IgG antibodies for Echinococcus granulosus by ELISA in years November 2011 - July 2013. Data was collected by attendant patients or suspected patients questionnaire information included (serial numbers; age; marital status; education and level of economic.

The assay used on all samples was an indirect Enzyme Linked Immunosorbent Assay (ELISA). Sensitivity and specificity of ELISA was calculated using histopathology as standard for those cases where histopathology results were available.

\section{Diagnosis}

Plain radiography, ultrasound, CT, and magnetic resonance imaging (MRI) are the most useful diagnostic tools for detection of hydatid cystic lesions [23]. CT and MRI in particular are highly sensitive for the diagnosis of hydatidosis and provide a complete lesion work-up [23,24]. Magnetic resonance Imaging (MRI scan) - MRI delineates the cyst capsule better than CT scan, as a low intensity on both T1 and T2 weighted images. However, CT scan is better in demonstrating mural calcifications, Laboratory and imaging data are usually sufficient to establish a reliable diagnosis but sometimes they are inconclusive [25]. Correct preoperative diagnosis is not always easy. Renal hydatid disease mimicked other diseases. The combination of clinical history, imaging studies, and serological and urine investigation yielded a reliable pretreatment diagnosis in only $50 \%$ of cases and a presumptive diagnosis in $71 \%$ [26,27]. Hypointense rim and multicystic appearance is distinctive in magnetic resonance imaging (MRI) scan which also delineates the anatomy well [28]. Clinically, renal hydatid cysts may remain asymptomatic for many years or may present with lumbar pain, hematuria, or intermittent fever. Most diagnoses are based on serology and imaging studies $[29,30]$.

CE diagnosis and monitoring firstly rely on imaging techniques. Ultrasonography (US) standardized classification of stage-specific cystic images has been issued by the WHO Informal Working Group on Echinococcosis (WHO-IWGE) for the diagnosis and the clinical management of CE [31]. Serologic tests such as direct hemagglutination, latex agglutination, and immunoelectrophoresis are widely used to confirm the diagnosis; however, they have low sensitivity and specificity [31,32]. Effective serological tests for CE diagnosis would be of great help to define and support cyst status and their evolution (active: CE1, CE2, and CE3b, transitional: CE3a, or inactive: CE4 and CE5) $[31,33]$. Early and proper diagnosis of CE can provide appropriate management and suitable treatment of the disease [34]. 


\section{Histopathology}

The technique was carried out on formalin fixed paraffin-embedded tissues (FFPT) from patients with histologically confirmed echinococcosis 2 sections $(5 \mu \mathrm{m})$ were prepared from tissue blocks and excess paraffin was removed. Sections were placed in oven for dewaxen for $10 \mathrm{~min}$ at $37{ }^{\circ} \mathrm{C}$. After dewaxen rehydration in $100 \%$, $90 \%, 80 \%$ and $70 \%$ ethanol was followed by $70 \%$ ethanol for $3 \mathrm{~min}$, then, water for $5 \mathrm{~min}$. then stained in Mayer haematoxyline for $5 \mathrm{~min}$. and bluing under tap water for $10 \mathrm{~min}$ and 3 minutes in eosin then rinsed 3 times in ascending alcohol and let to dry then mounted by DPX media.

\section{Ethical Consideration}

Ethical approval for the study was obtained from the Ethical Committee of federal ministry of health, and permission was provided from all hospitals where investigation conducted in addition and informed consent was obtained from each participant prior to interview.

\section{Statistical Analysis}

Data were analyzed by using the Statically Package for Social Science (SPSS).

\section{Results}

Out of 31 patients evaluated by serology for Echinococcosis during the study period, 7 (22.58\%) were seropositive. Liver was the most frequent site involved (24 patients; $77.77 \%$ ). Second organ the lung 5(16.13\%) bone $1(3.23 \%)$ and the optic $1(3.22 \%)$.Of the 29 patients who also underwent biopsy, all the seven patients with positive biopsy were seropositive, suggesting 100\% sensitivity. The specificity was $83.33 \%$, but this might be a lower estimate due to potentially high rate of falsenegative biopsies, as all the seropositive patients also had imaging features suggestive of disease. The positive and negative predictive values were $77.77 \%$ and $90.90 \%$, respectively.

A total of 32 patients were suspected of clinically and/or radiologically suffering from hydatid disease during the study period, and tested for IgG antibodies against hydatid disease. The mean age of all the patients was 45 years (range: 14-80 years). Highest numbers of cases were seen among age group of 31-60 years. Males and females constituted a nearly identical number of patients with suspected hydatid disease $(2$ and 2 , respectively). However, male patients were relatively younger compared to females, with a mean age of 38 and 45 years, respectively. Frequency distribution of age and gender is shown in Table 1.

Moreover, several studies have reported that the ultrasonography is more useful in diagnosing cystic Echinococcosis than CT and MRI $[52,53]$. When compared with CT scan, MRI has been shown to reproduce the findings of ultrasonography better [53].

\begin{tabular}{|c|c|c|c|c|c|c|c|}
\hline Organ & Gender & $<\mathbf{1 5}$ & $\mathbf{1 6 - 3 0}$ & $\mathbf{3 1 - 4 5}$ & $\mathbf{4 6 - 6 0}$ & $\mathbf{6 0}$ & Total \\
\hline \multirow{2}{*}{ Liver } & Male & & 1 & $2 / 1+\mathrm{ve}$ & $4 / 1+\mathrm{ve}$ & 4 & 11 \\
\cline { 2 - 8 } & female & $1+\mathrm{ve}$ & $2 / 1+\mathrm{ve}$ & 3 & 1 & 2 & 9 \\
\hline \multirow{2}{*}{ Lung } & Male & $1+\mathrm{ve}$ & & 1 & 3 & 3 & 8 \\
\cline { 2 - 8 } & Female & & & $1+\mathrm{ve}$ & & 1 & 2 \\
\hline \multirow{2}{*}{$\begin{array}{c}\text { Other } \\
\text { Origin }\end{array}$} & Male & & & & & 1 & 1 \\
\cline { 2 - 8 } & female & & & & $1+\mathrm{ve}$ & & 1 \\
\hline Total & & 2 & 3 & 7 & 9 & 11 & 32 \\
\hline
\end{tabular}

Table 1: Show hydatid cysts in different age group against gender.

Show the (Table 2) hydatid cysts were diagnostic by radiography finding majority of the cases were detected by CT scan there were 26 cases and 5 cases by USG and 1 case by MRI. CT is indicated in cases in which US fails due to patient-related difficulties (eg, obesity, excessive intestinal gas, abdominal wall deformities, previous surgery) or disease complications. CT has a high sensitivity and specificity for hepatic hydatid disease. CT may display the same findings as US. Cyst fluid usually demonstrates water attenuation. Calcification of the cyst wall or internal septa is easily detected at CT. Ultrasonography is the screening method of choice. It is currently the primary diagnostic technique and has diagnostic accuracy of $90 \%$.

Intravenous administration of contrast material is not necessary unless complications are suspected, especially infection and communication with the biliary tree (13).

\begin{tabular}{|c|c|c|c|c|c|}
\hline \multirow{2}{*}{ Origin } & Gender & USG & CT Scan & MRI & Total \\
\hline \multirow{2}{*}{ Liver } & Male & 3 & 8 & & 11 \\
\cline { 2 - 6 } & Female & 1 & 11 & & 12 \\
\hline \multirow{2}{*}{ Lung } & Male & 1 & 2 & & 3 \\
\cline { 2 - 6 } & Female & & 2 & & 2 \\
\hline \multirow{2}{*}{ Other Origins } & Male & & 1 & 1 & 2 \\
\cline { 2 - 6 } & Female & & 2 & & 2 \\
\hline Total & & 5 & 26 & 1 & 32 \\
\hline
\end{tabular}

Table 2: Table shows hydatid cysts in different radiography finding. 


\section{Examination of Cyst Fertility and Viability of Protoscoleces}

\section{Laboratory Protocol}

During the course of each operation, the hydatid fluid of the lesion was removed through a trocar and stored separately in sterile receptacles at room temperature. All samples were processed with the same protocol within 2 hours after their extraction in the Parasitology Laboratory. The first step consisted of sedimentation of the hydatid fluid in order to separate the hydatid gravel. Subsequently samples were taken and subjected to wet mount observation under a light microscope at different powers to evaluate the general characteristic of the protoscoleces, Finally, "vital" staining was applied to the preparations by staining with an aqueous solution of $0.1 \%$ eosin from which proceeded a count of protoscoleces and the description of the morphological aspects. And some of the biopsy fixed in $10 \%$ formalin.

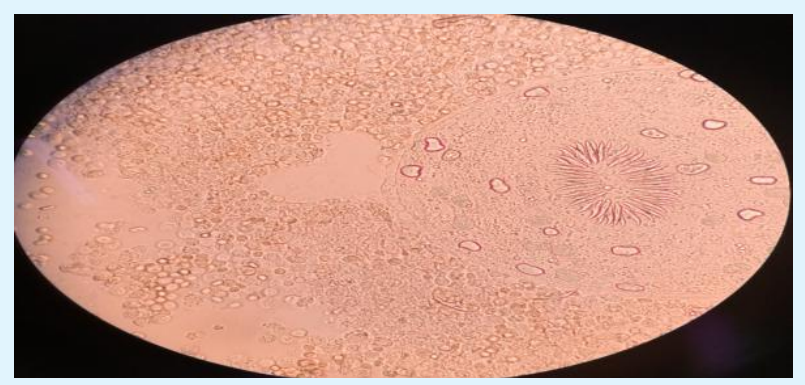

Figure 3: Wet mount observation: Significant mass of ovoid protoscoleces can be seen, with their normal structures and calcareous corpuscles intact.

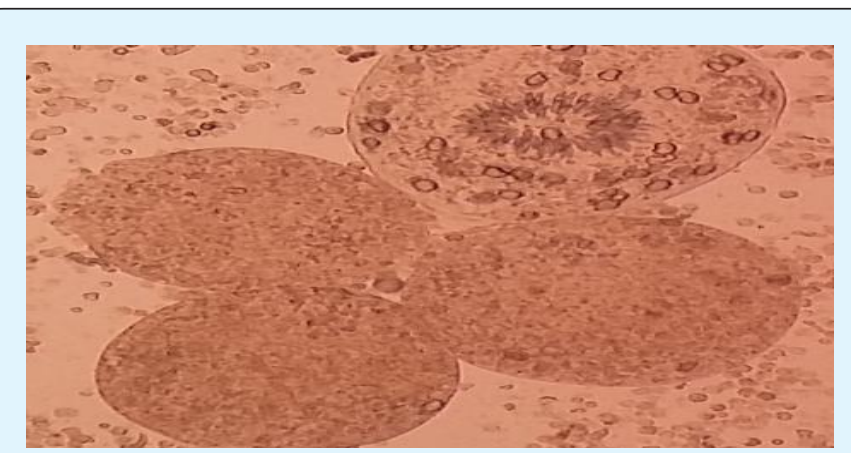

Figure 4: Wet mount observation: Significant mass of ovoid protoscoleces can be seen, with their normal structures and calcareous corpuscles intact.
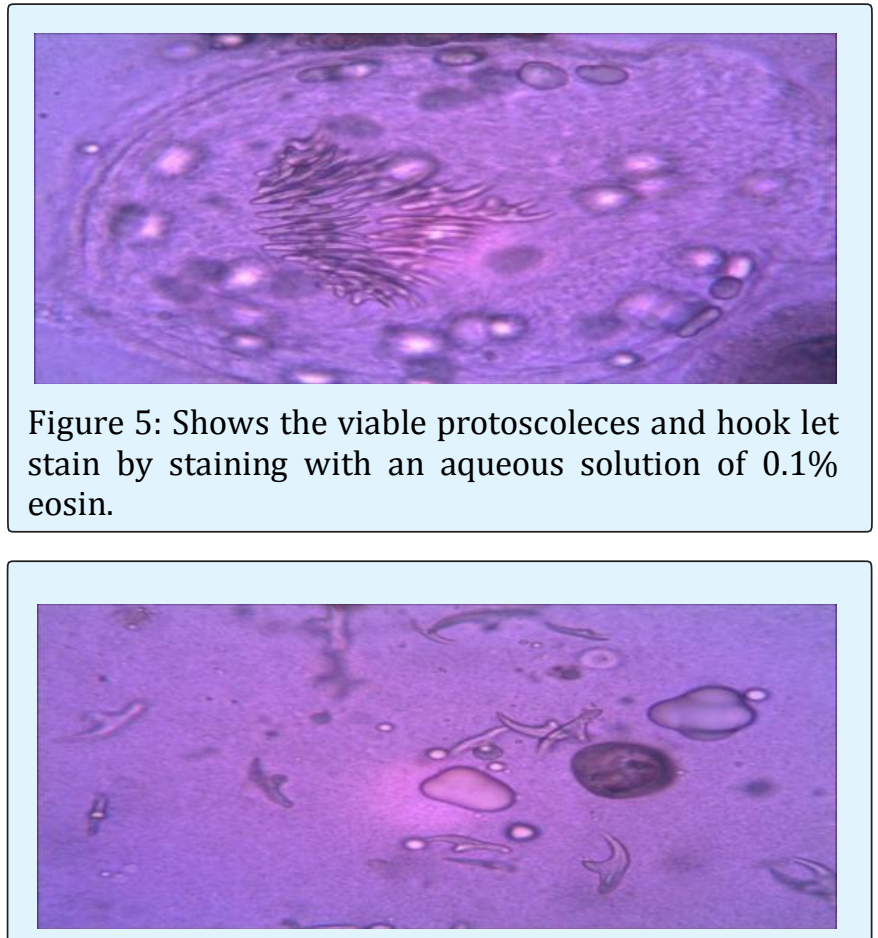

Figure 6: shows the viable hook let stain by staining with an aqueous solution of $0.1 \%$ eosin.

\section{Result of Histopathology}

Histopathological characteristics of cystic echinococcosis lesions in humans. Cystic echinococcal lesion in the liver (HE stain $\times 100$ ); laminated layer; germinal layer; protoscolesis.

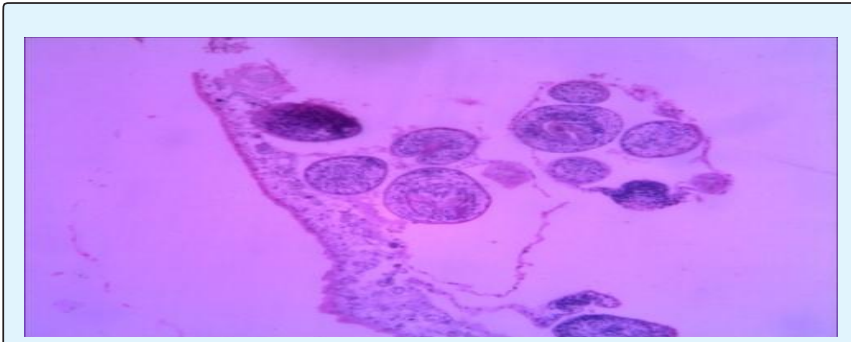

Figure 7: Section of specimen (1) liver tissue.

Diagnosis: Hydatid cyst

Microscopy: section of specimens show cyst wall composed of fibrous laminated layer with inner germinal layer with broad capsule and scolices surrounded by fibrous capsule. 


\section{Journal of Infectious Diseases \& Travel Medicine}

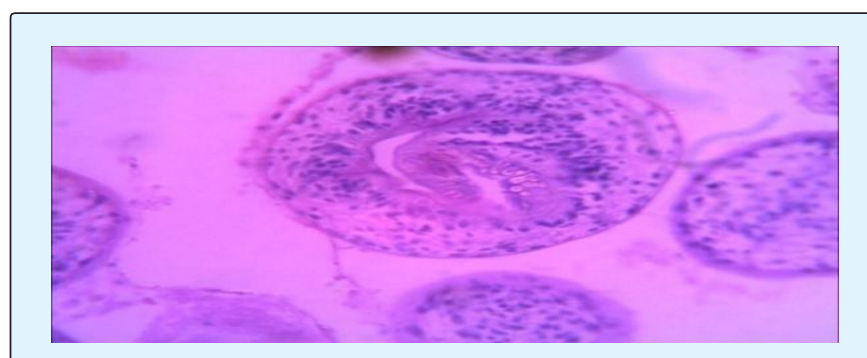

Figure 8: Section of specimen (2) lung tissue.

Diagnosis: Hydatid cyst

Microscopy: section of specimens show cyst wall composed of fibrous laminated layer with inner germinal layer with broad capsule and scolices surrounded by fibrous capsule

Specimen (3) Lung tissue

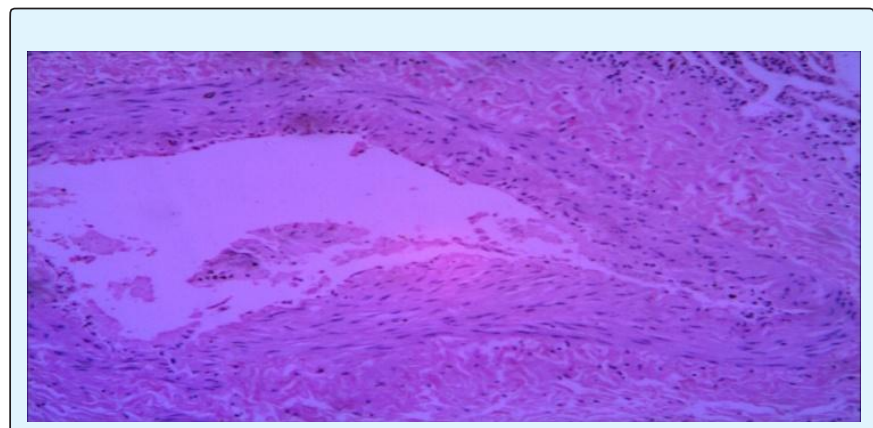

Figure 9: Section of specimen (3) lung tissue.

Diagnosis: Hydatid cyst.

Microscopy: section show lung tissue fibrovascular tissue with inflammatory granulation tissue. No Hydatid cyst

\section{Specimen (2): Lung tissue (4 \& 5)}

Diagnosis: pyogenic cavity

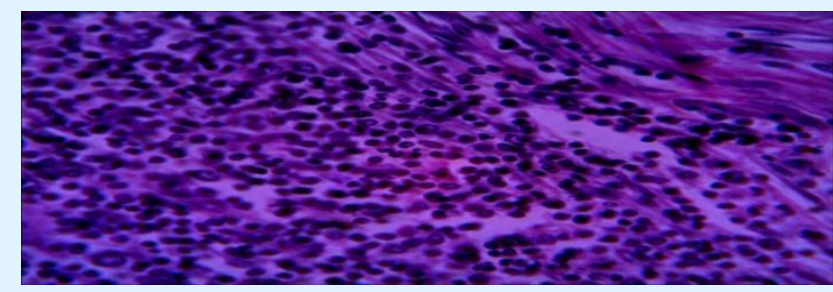

Figure 10: Section of specimen (4) lung tissue Microscopy: section show lung tissue with wall composed of inflammatory granulation tissue.

Microscopy: section show lung tissue with wall composed of inflammatory granulation tissue with suppuration. NO hydatid cyst.

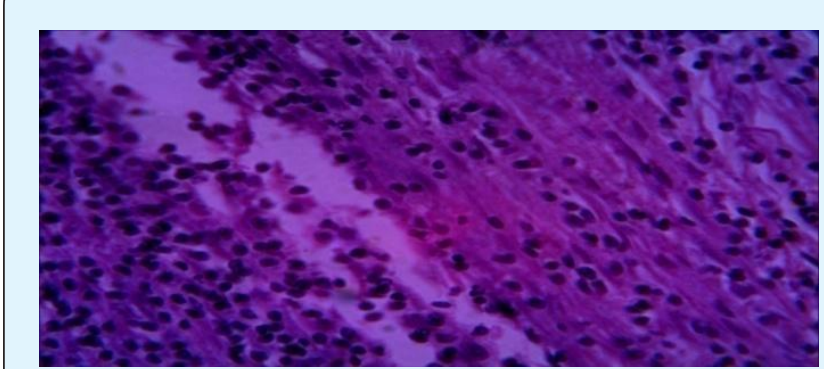

Figure 11: Section of specimen (5) lung tissue.

Diagnosis: pyogenic cavity

Microscopy: section show lung tissue with wall composed of inflammatory granulation tissue with suppuration. No hydatid cyst.

\section{Discussion}

This study was conducted on 31 patients of hydatid disease which included (35.48\%) serodiagnosis using (purified antigen) of Echinococcus granulosus cyst fluid male patients most of them being farmers 15(48.38\%) and female $16(51.61 \%)$ the maximum numbers of cases were housewives (table 1). The liver was most common affected organ 24(77.42\%), follow by lung 5(16.13\%), bone $1(3.23 \%)$ and optic $1(3.22 \%)$ (Table 2$)$. The higher rate of hepatic infection may be attributed to the fact that liver acts as primary filter in human body and lung is often thought to be the second filter.

Immunodiagnosis is an important tool for diagnosis of CE infection. Thus in addition to imaging techniques a reliable serodiagnosis improves prognosis for patients with cystic Echinococcus. The definitive diagnosis of cystic Echinococcusis by combination of CT scan or MRI with serological testing, the detection of circulating $\mathrm{E}$. granulosus in sera is less sensitive than antibody detection which remains the method of choice [35]. Insensitive and non-specific tests like Casoni intradermal test, complement fixation test, latex agglutination test and indirect haemagglutination test have been replaced by ELISA, indirect immunofluoresence antibody test, immunoelectrophoresis and immunoblotting [36]. Among these ELISA for detection of IgG antibodies is most commonly used. It considered being highly sensitive and specific in detecting anti-Echinococcus antibodies irrespective of the site of cyst localization [37]. The only place in the world that has the highest incidence and prevalence of hydatid disease is the Turkana district, due to the unique role that dogs play in the day-to-day life of 
their human owner her lungs, coughing and chest pain. Cysts can metastasize to other regions of the body as well.

As such, the Turkana have a whopping 7 to $10 \%$ echinococcosis prevalence rate and as many as $65 \%$ of canines can be infected [38]. Rates of infection are higher in the more northern, arid regions due to the considerable reliance upon livestock husbandry and a greater dependence upon dogs to clean items owing to the greater scarcity of water [15,39]. Higher prevalence of CE has been reported among nomadic Tibetan communities in China [40,41]. Earlier on pastoralism had been reported as risk factor for $\mathrm{CE}$ among pastoralists in Kasese district, Uganda [42,43]. This could be explained by the fact that among pastoralist, livestock keeping was the major activity. Dog-human-livestock interactions were intensive among these communities. Dogs were often kept for security reasons [44-46]. It has been consistently seen in various studies through the years and confirmed by our study that this disease in seen in young population who are in their active years.

The IgG levels had been estimated by the following Inhouse. In-house ELISA in which micro titration plate was coated with $2 \mu \mathrm{g} / 100 \mu \mathrm{l}$ of crude hydatid sheep antigen [37]. The DRG Echinococcus IgG ELISA Kit, a solid phase enzyme-linked immunosorbent assay (ELISA), in which microtiter wells as a solid phase are coated with Echinococcus crude antigen [47]. NovaLisa IgG ELISA Kit in which the microtiter wells as a solid phase are coated with Echinococcus crude antigen [48]. Ridascreen Echinococcus IgG Kit The micro test wells are coated with purified antigens i.e Specific IgG ELISA AgB [49].

Among these, ELISA for detection of IgG antibodies is most commonly used. It is considered to be highly sensitive and specific in detecting anti-Echinococcus antibodies irrespective of the site of cyst localization in similar study by Wattal, et al. [37]. Among purified antigens ELISA for IgG was the most sensitive (96.5\%) in comparison to our study which showed almost sensitivity of $100 \%$ [37]. These results are similar to those obtained by Kaddah, et al. [50]. The sensitivity and specificity of diagnosis of most of the tests varied considerably according to the nature, purity, and quality of antigen, according to the nature of the immunoglobulins (e.g. isotypes), and according to the sensitivity methodology chosen [51]. The reported high sensitivity of tests using purified antigens was produced in this study in our laboratory. Moreover, several studies have reported that the ultrasonography is more useful in diagnosing cystic Echinococcosis than CT and MRI [52,53]. When compared with CT scan, MRI has been shown to reproduce the findings of ultrasonography better Stojkovic, et al. [53]. Immunological investigations are always considered complimentary to imaging in diagnosing Echinococcosis due to lack of sensitivity and specificity. Antibody response in case with Echinococcosis infection largely depends on various factors such as the stage of disease, site of involvement, etc. Therefore false negative results are seen in cases of small cysts, calcified cysts, extra hepatic cysts and cysts in privileged sites $[54,55]$. Immunodiagnostic tests are also used for treatment monitoring in addition to primary diagnosis. IgG4 subset estimation is a good marker for treatment outcome; the levels decrease immediately after surgery and remain high in case of recurrent disease [35]. Hydatid cyst fluid (HCF), extracts from protoscolises, larval forms and adult worms are potential antigenic sources for the serological diagnosis of hydatid disease. Antigen $\mathrm{B}$ and antigen 5 are the most common constituents of HCF which are used for development of immunological tests. Among these, antigen $\mathrm{B}$ is a better antigen as compared to antigen 5 with respect to cross reactivity [56]. Detection of specific IgG subclasses have also shown to have diagnostic significance. Specific IgG4 iso type is shown to be associated with active disease and has been shown as a good prognostic marker in many studies $[54,56]$.

Histopathological examination of a suspected hydatid cyst is confirmatory, but is not routinely performed, as imaging and serological tests are usually diagnostic, and it has been considered that biopsy harbors the risk of anaphylaxis, and dissemination of disease. However, it may be of benefit in confirming diagnosis when other tests are ambiguous, or in cases with presentation at unusual sites. More than half of the patients in our study underwent histopathological evaluation, of which only 7 were reported positive. In comparison with histopathology, serology had $100 \%$ sensitivity, but a lower measured specificity of $81 \%$. However, it must be noted that all the 13 seropositive patients' had evidence of hydatid cyst on imaging, suggesting a possibly increased false negative rate on histopatholgical evaluation due to unexplained reasons.

\section{Conclusion}

Serology for detection of antibodies against hydatid disease is infrequently utilized. However, it is a simple test to confirm the diagnosis, and proved to be robust in our experience, with $100 \%$ sensitivity when compared with imaging and/or histopathology. The calculated specificity of $81.8 \%$ when compared to biopsy is likely an underestimation, as serology results correlated with imaging endings in all of the seropositive patients. 


\section{Journal of Infectious Diseases \& Travel Medicine}

\section{Competing Interests}

The authors declare that they have no competing interests.

\section{Author's Contribution}

AAMA was collected hydatid cysts, examined macroscopic and microscopic for the cysts, for the location, size, and is it fertile, also viability of protoscolsces and prepared the antigen. MHB was designed them and prepared the final manuscript, NTEO analyzed the final manuscript; MAM is director of the lab. Was helped with her experience, and AHG was competent and helped in the practical, HBO helped in the preparation of antigen.

\section{Acknowledgement}

Authors would like to thanks the colleagues who helped me in performing this study particularly to their cooperation for collecting hydatid cysts samples from hospitals in Khartoum state. This study was made possible by invaluable assistance provided by Mr. Hatim Babeker Osman is director of Poilo Lab. At the authors are very grateful to Miss: Aida Hassan Gaber and Mawheb Abdelmoneim, Mycology Department, University of Khartoum.

\section{References}

1. Lightowlers MW, Gauci CG, Chow C, Drew DR, Gauci SM, et al. (2003) Molecular and genetic characterisation of the host-protective oncosphere antigens of taeniid cestode parasites. Int J Parasitol 33(11): 1207-1217.

2. Moro P, Schantz PM (2009) Echinococcosis: a review. International Journal of Infectious Diseases 13(2): 125-133.

3. Khanfar N (2004) Hydatid disease: a review and update. Current Anaesthesia and Critical Care 15(3): 173-183.

4. WHO (2017) Echinococcosis, WHO Informal Working Group on Echinococcosis, Geneva.

5. Budke CM, Carabin H, Ndimubanzi PC, Nguyen H, Rainwater E, et al. (2013) A systematic review of the literature on cystic echinococcosis frequency worldwide and its associated clinical manifestations. Am J Trop Med Hyg 88(6): 1011-1027.
6. Budke CM, Deplazes P, Torgerson PR (2006) Global socioeconomic impact of cystic echinococcosis. Emerg Infect Dis 12(2): 296-303.

7. Thoma D, Romig T, Weible AK (2006) Echinococcus multilocularis a zoonosis of anthropogenic environments. Journal of Helminthology 80(2): 207212.

8. Jenkins DJ (2005) Hydatid control in Australia: where it began, what we have achieved and where to from here. International Journal for Parasitology 35(7): 733-740

9. McManus DP, Zhang W, Li J, Bartley PB (2003) "Echinococcosis". Lancet 362(9392): 1295-1304.

10. Grosso G, Gruttadauria S, Biondi A, Marventano S, Mistretta A (2015) Worldwide epidemiology of liver hydatidosis including the Mediterranean area. World J Gastroenterol 18(13): 1425-1437.

11. Sadjjadi SM (2006) Present situation of echinococcosis in the Middle East and Arabic North Africa. Parasitol Int 55: 197-202.

12. Eckert J Deplazes P (2004) Biological, epidemiological, and clinical aspects of echinococcosis, a zoonosis of increasing concern. Clinical Microbiology Reviews 17(1): 107-135.

13. Despommier DD, Gwadz RW, Hotez PJ, Knirsch CA (2006) Parasitic Diseases. 5th (Edn.), Apple Trees Production, LLC. Parasitology, New York 132(3): 449450.

14. King S, Hutchinson GW (2007) Hydatids-you, too, can be affected. Prime fact 475 NSW Department of Primary Industries pp: 1-9.

15. Romig T, Omer RA, Zeyhle E, Hüttner M, Dinkel A, et al. (2011) Echinococcosis in sub-Saharan Africa: emerging complexitiy. Vet Parasitol 181(1): 43-47.

16. Zlitini M, Ezzaoula k, Lebib H, Karray M, Kooli M, et al. (2001) Hydatid cyst of bone: diagnosis and treatment. World J Surg 25(1): 75-82.

17. Larrieu EJ, Frider B (2001) Human cystic echinococcosis: contributions to the natural history of the disease. Am J Trop Med Parasitol 95(7): 679-687.

18. Djuricic SM, Grebeldinger S, Kafka DI, Djan I, Vukadin M, et al. (2010) Cystic echinococcosis in children-the 


\section{Journal of Infectious Diseases \& Travel Medicine}

seventeen-year experience of two large medical centers in Serbia. Parasitol Int 59(2): 257-261.

19. Pierangeli NB, Soriano SV, Roccia I, Giménez J, Lazzarini LE, et al. (2007) Heterogeneous distribution of human cystic echinococcosis after a long-term control program in Neuquén, Patagonia Argentina. Parasitol Int 56(2): 149-155.

20. Tenguria RK, Naik MI (2014) Evaluation of human cystic echinococcosis before and after surgery and chemotherapy by demonstration of antibodies in serum. Annals of parasitology 60(4): 297-303.

21. Torgerson PR, Heath DD (2003) Transmission dynamics and control options for Echinococcus granulosus. Parasitology 127(7): 143-158.

22. Lahmar S, Debbek H, Zhang LH, McManus DP, Souissi A, et al. (2004) Transmission dynamics of the Echinococcus granulosus sheep-dog strain (G1 genotype) in camels in Tunisia. Vet Parasitol 121(12): 151-156.

23. Ataoglu H, Uckan S, Oz G, Altinörs N (2002) Maxillofacial hydatid cyst. J Oral Maxillofac Surg 60: 454-456.

24. Hafsa C, Ben Alaya T, Kriaa S, Jerbi S, Golli M, et al.( 2008) Maxillo-facial location of hydatic cyst. Ann Otolaryngol Chir Cervicofac 125: 160-163.

25. Brunetti E, Kern P, Vuitton DA (2010) Expert consensus for the diagnosis and treatment of cystic and alveolar echinococcosis in humans. Acta Tropica 114(1): 1-16.

26. Ameur A, Lezrek M, Boumdin H, Touiti D, Abbar M, et al. ( 2002) Hydatid cyst of the kidney based on a series of 34 cases. Prog Urol 12(3): 409-414.

27. Gogus C, Safak M, Baltaci S, Turkjolmez Z ((2003) Isolated renal hydatidosis: Experience with 20 cases. J Urol 169(1): 186-189.

28. Turgut AT, Ödev K, Kabaalioğlu A, Bhatt S, Dogra VS (2009) Multitechnique evaluation of renal hydatid disease. AJR Am J Roentgenol 192(2): 462-467.

29. Gharde P, Wagh DD, Patil A (2012) Left renal hydatid cyst presenting as hematuria and macroscopic hydatiduria since last ten years. Trop Parasitol 2(1): 58-60.
30. Shah KJ, Ganpule AP, Desai MR (2009) Isolated renal hydatid cyst managed by laparoscopic transperitoneal nephrectomy. Indian J Urol 25(4): 531-533.

31. Bouckaert MM, Raubenheimer EJ, Jacobs FJ (2000) Maxillofacial hydatid cysts. Oral Surg Oral Med Oral Pathol Oral Radiol Endod 89(3): 338-342.

32. Katilmiş H, Oztürkcan S, Ozdemir I, AdadanGüvenç I, Ozturan S (2007) Primary hydatid cyst of the neck. Am J Otolaryngol 28(3): 205-207.

33. Hosch W, Junghanss T, Stojkovic M, Brunetti E, Heye $\mathrm{T}$, et al. (2008) Metabolic viability assessment of cystic echinococcosis using high-field 1H MRS of cyst contents. NMR Biomed 21(7): 734-54.

34. Zhang W, Wen H, Li J, Lin R, McManus DP (2012) Immunology and immunodiagnosis of cystic echinococcosis: an update. Clin Dev Immunol 2012: 101895.

35. Zhang W, Li J, McManus DP (2003) Concepts in immunology and diagnosis of hydatid disease. Clin Microbiol Rev 16(1): 18-36.

36. Lightowlers MW, Gottstein B, Thompson RCA, Lymbery AJ (1995) Echinococcosis/hydatidosis: antigens, immunological and molecular diagnosis. Echinococcus and hydatid disease. CAB International Wallingford, UK, pp: 355-410.

37. Wattal C, Malla N, Khan IA, Agarwal SC (1986) Comparative evaluation of enzyme linked immunosorbent assay for the diagnosis of pulmonary Echinococcosis. J Clin Microbiol 24(1): 41-46.

38. Magambo J, Njoroge E, Zeyhle E (2006) Epidemiology and control of echinococcosis in sub-Saharan Africa. Parasitol Int 193-195.

39. Cooney RM, Flanagan KP, Zehyle E (2004) Review of surgical management of cystic hydatid disease in a resource limited setting: Turkana, Kenya. Euro J of Gastroenterology and Hepatology 16(11): 1233-1236.

40. Schantz PM, Wang H, Qiu J, Liu FJ, Saito E, et al. (2003) Echinococcosis on the Tibetan Plateau: Prevalence and risk factors for cystic and alveolar echinococcosis in Tibetan populations in Qinghai Province, China. Parasitology 127: 109-120. 


\section{Journal of Infectious Diseases \& Travel Medicine}

41. Wang Q, Huang Y, Huang L, Yu W, He W, et al. (2014) Review of risk factors for human echinococcosis prevalence on the Qinghai-Tibet Plateau, China: A prospective for control options. Infect Dis Poverty 3(1): 3 .

42. Nyakarahuka L, Lalobo-Oryema M, Kankya C, Siefert L, Ocaido M, et al. (2012) Knowledge, attitude and practices towards cystic echinococcosis in pastoral communi-ties in Kasese district. Adv Trop Med Public Health Int 2: 32-39.

43. Luke N, Francis E, MichealL O, Clovice K, Ludwig S, et al. (2013) A survey of potential risk factors associatedwith cystic echinococcosis in pastoral communities in Kasese distract, Uganda. Adv Trop Med Public Health Int 3: 10-24.

44. Li D, Gao Q, Liu J, Feng Y, Ning W, et al. (2015) Knowledge, attitude, and practices (KAP) and risk factorsanalysis related to cystic echinococcosis among residents in Tibetan communities, Xiahe County, Gansu Province, China. Acta Trop 147: 17-22.

45. Yang YR, Ellis M, Sun T, Li Z, Liu X, et al. (2006) Unique family clustering of human echinococcosis cases ina Chinese community. Am J Trop Med Hyg 74(3): 487-494.

46. Oba P, Ejobi F, Omadang L, Chamai M, Okwi AL, et al. (2016) Prevalence and risk factors of Echinococcus granulosus infection in dogs in Moroto and Bukedea districts in Uganda. Trop Anim Health Prod 48(2): 249-254.

47. (2012) DRG Echinococcus granulosus IgG (EIA-3472). Manual 1-12.

48. Nova Lisa TM ECHG 0130 Echinococcus IgG ELISA Kit manual. 1-26.
49. Ridascreen Echinococcus IgG (R-Biopharm AG. Landwehrstr, 54, D-64293 Darmstadt, Germany). 110.

50. Kaddah $\mathrm{MH}$, Maher KM, Hassanein HI, Farrag AL, Shaker ZA, et al. (1992) Evaluation of different immunodiagnosis techniques for diagnosis of hydatidosis in Egypt. J Egypt Soc Paraitol 22(3): 653665.

51. Gottstein B (1992) Molecular and immunological diagnosis of Echinococcosis. Clin Microbiol Rev 5(3): 248-261.

52. Wuestenberg J, Gruener B, Oeztuerk S, Mason RA, Haenle MM, et al. (2014) Diagnostics in cystic echinococcosis: serology versus ultrasonography. Turk J Gastroenterol 25(4): 398-404.

53. Stojkovic M, Rosenberger K, Kauczor HU, Junghanss T, Hosch W (2012) Diagnosing and staging of cystic echinococcosis: how do CT and MRI perform in comparison to ultrasound? PloS Negl Trop Dis 6(10): e1880.

54. Manzano-Román R, Sánchez-Ovejero C, HernándezGonzález A, Casulli A, Siles-Lucas M (2015) Serological diagnosis and follow up of human cystic echinococcosis: a new hope for the future? Biomed Res Int 2015: 428205.

55. Florea A, Vlad L, Cozma V, Coroiu Z (2009) Serological diagnosis of cystic echinococcosis by the ELISA technique, in the cases hospitalized in the surgical clinic no. III and internal medicine no. III of ClujNapoca. Sci Parasitol 12(3): 167-171.

56. Sarkari B, Rezaei Z (2015) Immunodiagnosis of human hydatid disease: where do we stand? World J Methodol 5(4): 185-195.

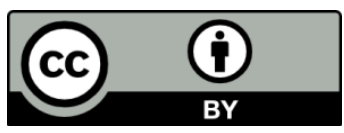

